\title{
Bunch-by-bunch measurement of transverse coherent beam-beam modes in the Fermilab Tevatron collider
}

\author{
Giulio Stancari* and Alexander Valishev \\ Fermi National Accelerator Laboratory, P.O. Box 500, Batavia, Illinois 60510, USA
}

(Received 21 April 2011; published 3 April 2012)

\begin{abstract}
A system for bunch-by-bunch detection of transverse proton and antiproton coherent oscillations in the Tevatron is described. It is based on the signal from a single beam-position monitor located in a region of the ring with large amplitude functions. The signal is digitized over a large number of turns and Fourier analyzed off-line with a dedicated algorithm. To enhance the signal, band-limited noise is applied to the beam for about $1 \mathrm{~s}$. This excitation does not adversely affect the circulating beams even at high luminosities. The device has a response time of a few seconds, a frequency resolution of $1.6 \times 10^{-5}$ in fractional tune, and it is sensitive to oscillation amplitudes of $60 \mathrm{~nm}$. It complements Schottky detectors as a diagnostic tool for tunes, tune spreads, and beam-beam effects. Measurements of coherent mode spectra are presented to show the effects of betatron tunes, beam-beam parameter, and collision pattern, and to provide an experimental basis for beam-beam numerical codes. Comparisons with a simplified model of beam-beam oscillations are also described.
\end{abstract}

DOI: 10.1103/PhysRevSTAB.15.041002

PACS numbers: 29.20.db, 29.27.-a

\section{INTRODUCTION}

In particle colliders, each beam experiences nonlinear forces when colliding with the opposing beam. A manifestation of these forces is a vibration of the bunch centroids around the closed orbit. These coherent beam-beam oscillation modes were observed in several lepton machines, including PETRA, TRISTAN, LEP, and VEPP-2M [1-4]. Although their observation in hadron machines is made more challenging by the lack of strong damping mechanisms to counter external excitations, they were seen both at the ISR and at RHIC [5-9]. Originally, one motivation for the study of coherent beam-beam modes was the realization that their frequencies may lie outside the incoherent tune distribution, with a consequent loss of Landau damping [10]. The goal of the present research is to develop a new diagnostic tool to estimate bunch-by-bunch tune distributions, to assess the effects of Gaussian electron lenses for beam-beam compensation [11-14], and to provide an experimental basis for the development of beam-beam numerical codes.

The behavior of colliding bunches is analogous to that of a system of oscillators coupled by the beam-beam force. In the simplest case, when 2 identical bunches collide headon in one interaction region, 2 normal modes appear: a $\sigma$

\footnotetext{
*Corresponding author. stancari@fnal.gov

On leave from Istituto Nazionale di Fisica Nucleare (INFN), Sezione di Ferrara, Italy.

Published by the American Physical Society under the terms of the Creative Commons Attribution 3.0 License. Further distribution of this work must maintain attribution to the author(s) and the published article's title, journal citation, and DOI.
}

mode (or 0 mode) at the lattice tune, in which bunches oscillate transversely in phase, and a $\pi$ mode, separated from the $\sigma$ mode by a shift of the order of the beam-beam parameter, in which bunches are out of phase. In general, the number, frequency, and amplitude of these modes depend on the number of bunches, on the collision pattern, on the tune separation between the two beams, on transverse beam sizes, and on relative intensities. Coherent beam-beam modes have been studied at several levels of refinement, from analytical linear models to fully 3-dimensional particle-in-cell calculations [1,9,15-21].

In the Tevatron, 36 proton bunches (identified as P1-P36) collide with 36 antiproton bunches (A1-A36) at the centerof-momentum energy of $1.96 \mathrm{TeV}$. There are 2 head-on interaction points (IPs), corresponding to the CDF and the DZero experiments. Each particle species is arranged in 3 trains of 12 bunches each, circulating at a revolution frequency of $47.7 \mathrm{kHz}$. The bunch spacing within a train is $396 \mathrm{~ns}$, or $2153-\mathrm{MHz}$ rf buckets. The bunch trains are separated by $2.6-\mu \mathrm{s}$ abort gaps. The synchrotron frequency is $34 \mathrm{~Hz}$, or $7 \times 10^{-4}$ times the revolution frequency. The machine operates with betatron tunes near 20.58.

The betatron tunes and tune spreads of individual bunches are among the main factors that determine beam lifetimes and collider performance. They are affected by head-on and long-range beam-beam interactions. Three systems are currently used in the Tevatron to measure incoherent tune distributions: the 21.4-MHz Schottky detectors, the 1.7-GHz Schottky detectors, and the direct diode detection base band tune (3D-BBQ). The latter two can be gated on single bunches. Detection of transverse coherent modes can complement these three systems because of its sensitivity, bunch-by-bunch capability, high frequency resolution, and fast measurement time. 
The basis for the measurement technique was presented in Ref. [22], and preliminary results can be found in Refs. [23-25]. Several improvements, mainly in the data analysis, were implemented and presented in a concise report [26]. In this paper, we describe the detection technique in detail. We also present a wide set of measurements illustrating the performance of the device and the response of the coherent mode spectra to various experimental conditions, such as betatron tune separation, beam-beam parameter, and collision pattern.

\section{MODELING}

The basic features of transverse coherent oscillations can be described by a simple model. In the Tevatron, these oscillations are substantially nonlinear due to the properties of the lattice and of the beam-beam force. Hence, the rigid bunch approximation cannot provide an accurate view of the coherent mode spectrum. However, this approximation can be used for qualitative analysis of the expected beam-beam mode tunes and their dependence on the betatron tunes $Q$ and the beam-beam parameter per interaction point $\xi$.

We use a simple matrix formalism to compute the eigenmode tunes of the system of colliding bunches. Besides employing the rigid bunch approximation, one more simplification is used. The complete description of the system would require modeling the interaction of 72 bunches at 138 collision points. The analysis of such a system can be quite complex. Observations and analytical estimates show that the difference in tunes between individual bunches is small compared to the beam-beam tune shift. Thus, as a first approximation, it is possible to neglect long-range interactions. This limits the system to 6 bunches $(3$ in each beam) colliding at two head-on interaction points. In the following discussion, we limit betatron oscillations to one degree of freedom. Because the system has 3-fold symmetry, the 1-turn map transporting the 12-vector of dipole moments and momenta of the system of 6 bunches can be expressed as follows:

$$
M=M_{\mathrm{BB} 3} M_{\mathrm{T} 3} M_{\mathrm{BB} 2} M_{\mathrm{T} 2} M_{\mathrm{BB} 1} M_{\mathrm{T} 1},
$$

where $M_{\mathrm{TN}}(N=1,2,3)$ are the $2 \times 2$ block-diagonal $12 \times 12$ matrices transporting phase space coordinates through the accelerator arcs, and $M_{\mathrm{BBN}}$ are the matrices describing thin beam-beam kicks at the IPs. Although there are only 2 interactions per bunch, 3 collision matrices are used to describe a 1-turn map of the system of 6 bunches. This construction represents the time propagation of the bunch coordinates through one turn with break points at the $\mathrm{CDF}$ (B0), D0, and F0 locations in the machine. If on a given step the bunch is at $\mathrm{B} 0$ or $\mathrm{D} 0$, its momentum coordinate is kicked according to the distance between the centroids of this bunch and of the opposing bunch. If the bunch is at F0 (1/3 of the circumference from B0 and D0), where the beams are separated, its momentum is unchanged. For example, the matrix describing the interaction of proton bunch 1 with antiproton bunch 2 at $\mathrm{CDF}$ and proton bunch 3 with antiproton bunch 3 at DZero has the following form:

$$
M_{\mathrm{BB} 1}=\left(\begin{array}{ccccc}
1 & 0 & 0 & 0 & 0 \\
-2 \pi \xi^{p} / \beta & 1 & 0 & 0 & 0 \\
0 & 0 & 1 & 0 & 0 \\
0 & 0 & 0 & 1 & 0 \\
0 & 0 & 0 & 0 & 1 \\
0 & 0 & 0 & 0 & -2 \pi \xi^{p} / \beta \\
0 & 0 & 0 & 0 & 0 \\
0 & 0 & 0 & 0 & 0 \\
0 & 0 & 0 & 0 & 0 \\
2 \pi \xi^{a} / \beta & 0 & 0 & 0 & 0 \\
0 & 0 & 0 & 0 & 0 \\
0 & 0 & 0 & 0 & 2 \pi \xi^{a} / \beta
\end{array}\right.
$$

Here, $\xi^{p}$ and $\xi^{a}$ are the beam-beam parameters for protons and antiprotons, and $\beta$ is the amplitude function at the IP. The Yokoya factor [16,27] is considered to be equal to 1 . The eigentunes of the 1-turn map are then computed numerically.

This model provides a quick estimate of the expected values of the coherent beam-beam mode tunes for a given

$\left.\begin{array}{ccccccc}0 & 0 & 0 & 0 & 0 & 0 & 0 \\ 0 & 0 & 0 & 2 \pi \xi^{p} / \beta & 0 & 0 & 0 \\ 0 & 0 & 0 & 0 & 0 & 0 & 0 \\ 0 & 0 & 0 & 0 & 0 & 0 & 0 \\ 0 & 0 & 0 & 0 & 0 & 0 & 0 \\ 1 & 0 & 0 & 0 & 0 & 2 \pi \xi^{p} / \beta & 0 \\ 0 & 1 & 0 & 0 & 0 & 0 & 0 \\ 0 & 0 & 1 & 0 & 0 & 0 & 0 \\ 0 & 0 & 0 & 1 & 0 & 0 & 0 \\ 0 & 0 & 0 & -2 \pi \xi^{a} / \beta & 1 & 0 & 0 \\ 0 & 0 & 0 & 0 & 0 & 1 & 0 \\ 0 & 0 & 0 & 0 & 0 & -2 \pi \xi^{a} / \beta & 1\end{array}\right)$.

set of machine and beam parameters. The model cannot be used for accurate calculation of the relative amplitude of these modes, which is determined by nonlinear effects such as Landau damping. For the case of weak nonlinearities, this approach allows one to determine the mode amplitudes by computing the projection of mode eigenvectors on the excitation vector [4]. In the case of the Tevatron 


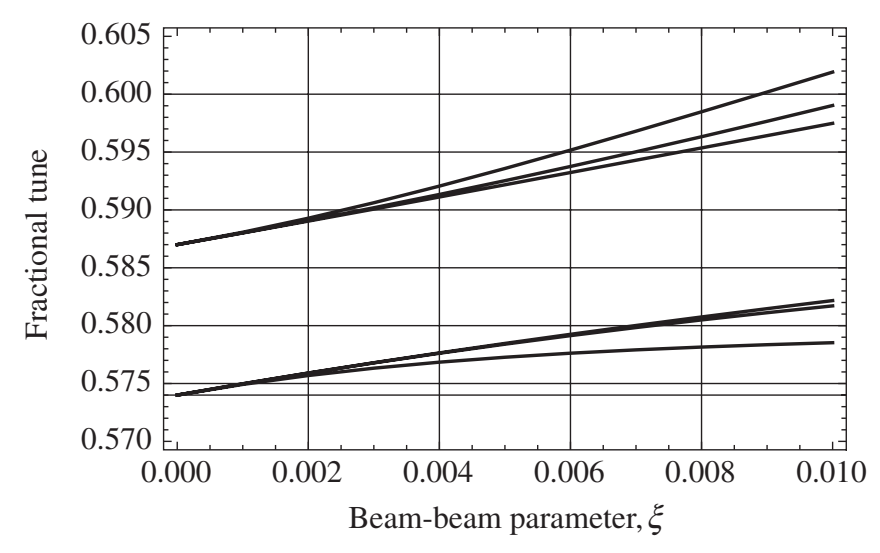

FIG. 1. Coherent mode tunes vs beam-beam parameter calculated with the linearized model; $Q^{p}=0.587, Q^{a}=0.574$, $\xi=\xi^{p}=\xi^{a}$.

experiments described below, this is not straightforward because a wideband noise source was used to excite the beam motion.

In Fig. 1, an example of the dependence of the 6 eigenfrequencies on the beam-beam parameter per IP is presented. As one would expect, at small values of $\xi$ (uncoupled oscillators) the mode frequencies approach the bare lattice tunes; in this case, 0.587 for protons and 0.574 for antiprotons. When the total beam-beam parameter exceeds the difference between the lattice tunes, the modes are split and their symmetry approaches that of the conventional $\sigma$ and $\pi$ modes. The parameters of this calculation are taken to resemble those of the beginning of the Tevatron Store 7754, when the beam-beam parameter was $\xi=\xi^{a}=\xi^{p}=0.01$. A comparison with data is given in Sec. V (Fig. 13).

\section{APPARATUS}

The system for the detection of transverse coherent modes (Fig. 2) is based on the signal from a single vertical beam-position monitor (BPM) located near the CDF interaction point, in a region where the vertical amplitude function at collisions is $\beta_{y}=880 \mathrm{~m}$. The BPM is a stripline pickup, with two plate outputs $(A$ and $B$ ) for each of the two counterpropagating beams. The proton outputs are split: half of the signal is sent to the Tevatron BPM readout and orbit stabilization circuits; the other half is used by the present system. Antiproton signals are about a factor 3 weaker and are usually not used for orbit feedback, so the splitter is not necessary and the full signal can be analyzed. Switching between proton and antiproton signals presently requires physically swapping cables.

In the Tevatron, protons and antiprotons share a common vacuum pipe. Outside of the interaction regions, their orbits wrap around each other in a helical arrangement. Therefore, bunch centroids can be several millimeters away from the BPM's electrical axis. Typically, the

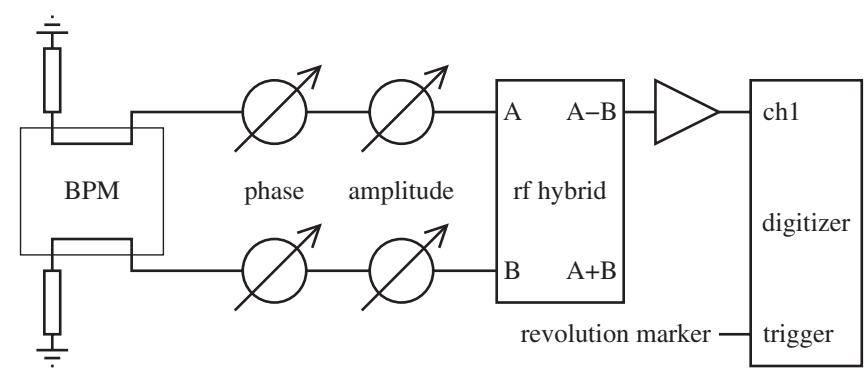

FIG. 2. Schematic diagram of the apparatus.

peak-to-peak amplitude of the proton signal is $10 \mathrm{~V}$ on one plate and $5 \mathrm{~V}$ on the other, whereas the signal of interest is of the order of a few millivolts. For this reason, it is necessary to equalize the $A$ and $B$ signals to take advantage of the full dynamic range of the digitizer. Equalization also reduces false transverse signals due to trigger jitter, as discussed below. The phase and attenuation of each signal is manually adjusted by minimizing the $A-B$ output of the rf hybrid circuit. If necessary, finetuning is done by displacing the beam with a small orbit bump. Figure 3 shows an example of $A$ and $B$ signals after equalization and the $A-B$ output of the hybrid. Orbits at collisions are stable over a time scale of weeks, and this manual adjustment does not need to be repeated often. To automate the task in the case of changing orbits and intensities (e.g., for observations at top energy between the low-beta squeeze and initiating collisions, or for observing both proton and antiproton bunches), a circuit board is being designed with self-calibrating gains and offsets.

The difference signal from the hybrid is amplified by $23 \mathrm{~dB}$ and sent to the digitizer. We use a 1-channel, 1-V full range, 10-bit digitizer (Agilent Acqiris series) with timeinterleaved analog-to-digital converters (ADCs). It can sample at $8 \mathrm{GS} / \mathrm{s}$ and store a maximum of $1024 \mathrm{MS}$ or 125000 segments. (Because of a firmware problem, only half of the segments were used in the experiments described below.) The 47.7-kHz Tevatron revolution marker is used as a trigger, so we will refer to "segments" or "turns" interchangeably. Typically, we sample at $8 \mathrm{GS} / \mathrm{s}$ (sample period of $125 \mathrm{ps}$ ), which corresponds to 150 slices for each 19-ns rf bucket. At this sampling rate, one can record waveforms of 1 bunch for 62500 turns, 2 bunches for 52707 turns, or 12 bunches for 12382 turns, depending on the measurement of interest. A C++ program running on the front-end computer controls the digitizer settings, including its delay with respect to the Tevatron revolution marker.

Data is written in binary format. The output contains the raw ADC data together with the trigger time stamps and the delay of the first sample with respect to the trigger. Timing information has an accuracy of about $15 \mathrm{ps}$, and it is extremely important for the synchronization of samples from different turns. 

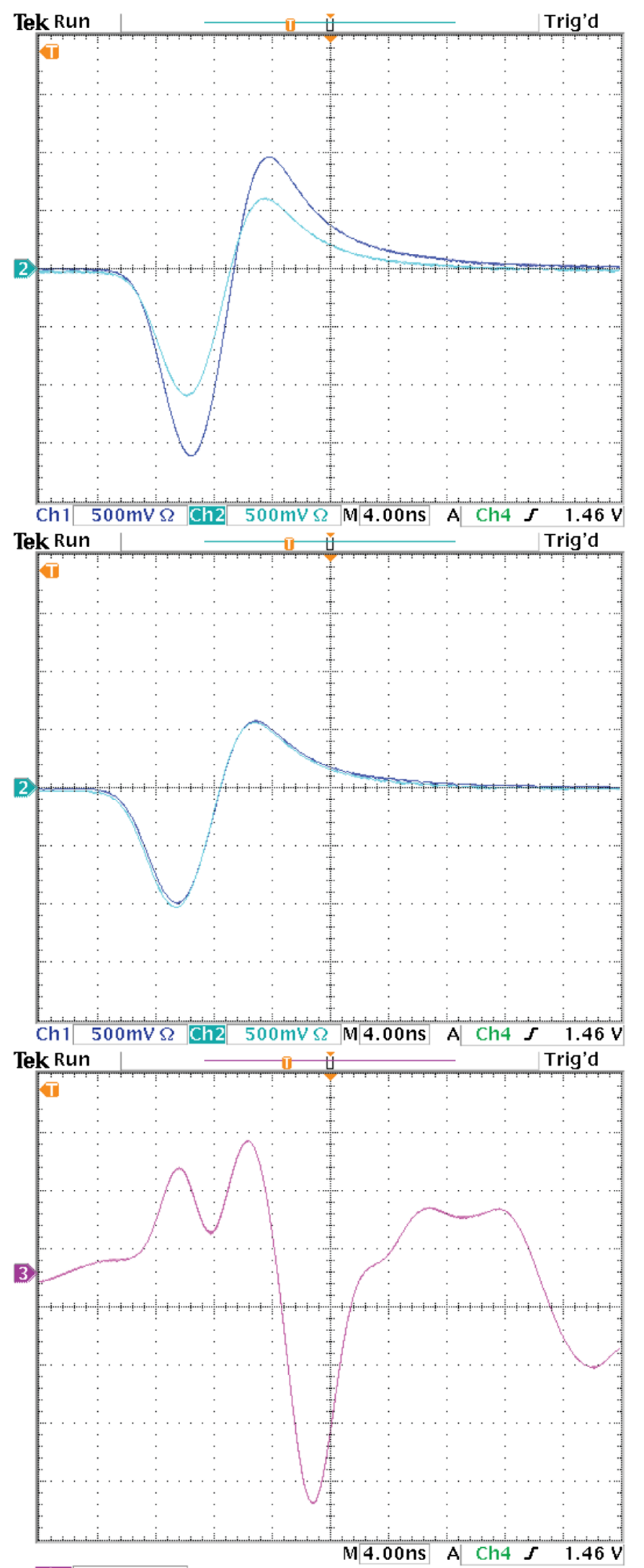

Ch3 $20.0 \mathrm{mV \Omega}$

FIG. 3. BPM signals $A$ (blue) and $B$ (cyan) for an antiproton bunch (top); same signals after equalization (center); $A-B$ output of the hybrid circuit after amplification (magenta, bottom).
To enhance the signal, the beam is excited with a few watts of band-limited noise ("tickling") for about $1 \mathrm{~s}$ during the measurement. The measurement cycle consists of digitizer setup, tickler turn-on, acquisition start, tickler turn-off, and acquisition stop. The cycle takes a few seconds. The procedure is parasitical and it does not adversely affect the circulating beams, even at the beginning of regular collider stores, with luminosities around $3.5 \times 10^{32}$ events $/\left(\mathrm{cm}^{2} \mathrm{~s}\right)$. When repeating the procedure several times, the Schottky monitors may show some activity, but no beam loss is observed.

\section{DATA ANALYSIS}

Data is analyzed off-line using the multiplatform, open-source R statistical package [28]. The distribution of differences between trigger time stamps from consecutive turns yields the average revolution frequency (47713.11 Hz at $980 \mathrm{GeV})$. From it, the nominal or "ideal" trigger time stamps for each turn are calculated. The distribution of trigger offsets, i.e., the differences between measured and nominal time stamps, is a measure of the jitter in the revolution marker (Fig. 4, top left). The root mean square of the distribution is usually less than $0.2 \mathrm{~ns}$. The delay between trigger time and the time stamp of the first sample is also recorded with an accuracy of $15 \mathrm{ps}$. An offset distribution is shown in Fig. 4 (top right). As expected for uncorrelated revolution period and sampling clock, the offsets do not exceed the sampling period (125 ps, in this case) and their distribution is flat. The sum of trigger offset and first-sample delay is the correction by which each sample in a segment is to be shifted in time to be aligned with the other segments. For each turn and each bunch, the signal is interpolated with a natural spline and shifted in time according to this correction. One undesirable effect of this synchronization algorithm is that a few slices (usually not more than 3 ) at each edge of the bucket become unusable, as they cannot be replaced with real data. The synchronization of turns is extremely important, as the jitter in trigger time translates into a false transverse oscillation where the difference signal has a slope. If the BPM plates are not perfectly balanced, jitter of even a fraction of a nanosecond can raise the noise floor by several decibels and compromise the measurement.

Figure 4 shows the distribution of digitized data for each slice in an antiproton bunch after synchronization (center left) and after subtracting the average for each slice (center right). Each slice corresponds to $125 \mathrm{ps}$. At the bottom of Fig. 4 is the difference signal (proportional to beam position) over the course of a measurement (52 707 turns in this case). Bunch oscillations are dominated by low-frequency beam jitter attributable to mechanical vibrations [29]. The range of amplitudes is inferred from comparisons with the regular Tevatron BPM system and corresponds to about $\pm 25 \mu \mathrm{m}$. This low-frequency jitter does not affect the measurements of coherent beam-beam modes directly, 

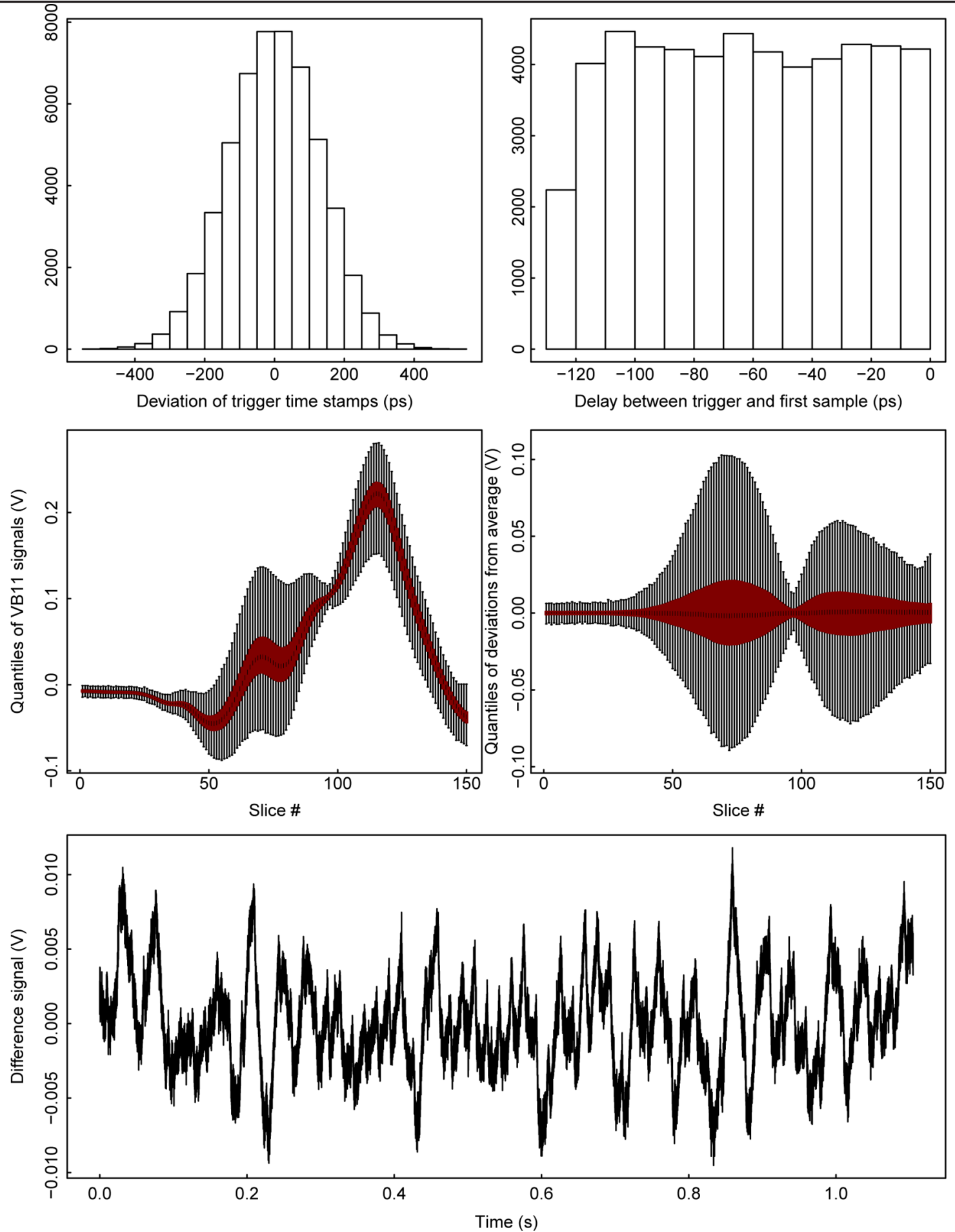

FIG. 4. Summary plots for one sample data set (Store 7754 at 21:41): difference between recorded trigger time and nominal revolution time (top left); recorded offset between trigger time stamp and first sample (top right); quantiles (minimum, 25\%-75\% in red, maximum) of digitized signals over all 52707 turns, for each slice (center left); quantiles of digitized signals after subtracting each slice's average; average difference signal for the signal slices (41-95 and 99-147) over the course of the measurement.

but it reduces the available dynamic range. A high-pass filter and more amplification may be employed to improve the system.

For each bunch, the signal of each individual slice vs turn number is Fourier transformed. Frequency resolution is determined by the number of bins in the fast Fourier transform (FFT) vector and it is limited to 62500 turns, corresponding to $1.6 \times 10^{-5}$ of the revolution frequency or $0.8 \mathrm{~Hz}$. The data is multiplied by a Slepian window of rank 2 to confine leakage to adjacent frequency bins and 
suppress it below $10^{-5}$ in farther bins [30]. When the full frequency resolution is not needed, the FFT vectors are overlapped by about $1 / 3$ of their length to reduce data loss from windowing, and the resulting spectral amplitudes are averaged.

Calculations take about $20 \mathrm{~s}$ per bunch for 62500 turns and 150 slices per bunch on a standard laptop computer (Apple MacBook running Mac OS X 10.5.8 with 2.4-GHz Intel Core 2 Duo processor and 4 GB of RAM). Processing time is dominated by the synchronization algorithm.

The noise level is estimated by observing the spectra without beam. The spectra show a few sharp lines in all slices. These lines are attributed to gain and offset differences between the time-interleaved ADCs themselves and to timing skew of their clocks [31-33]. The same spurious lines are also present in the Fourier spectrum of the time stamps, and this corroborates their attribution to digitizer noise. To improve the signal-to-noise ratio, and to suppress backgrounds unrelated to the beam such as the spurious lines from the digitizer, a set of signal slices (near the signal peaks) and a set of background slices (before the arrival of the bunch) are defined. Amplitude spectra are computed for both signal and background slice sets, and their ratio is calculated. The ratios are very clean, with some additional variance at the frequencies corresponding to the narrow noise spikes. Results are presented in terms of these signal-to-background amplitude ratios.

Figure 5 shows an example of analyzed antiproton data, in two regions of the frequency spectrum: a low-frequency region with the horizontal axis expressed in hertz (top two plots) and a high-frequency region, in terms of the revolution frequency or fractional tune. The 2-dimensional color plots show the amplitude distribution for each of the 150 125 -ps slices in logarithmic scale. In this example, the signal slices are numbers 41-95 and 99-147. They are defined as the ones for which the amplitude is above $10 \%$ of the range of amplitudes (see also Fig. 4, center right). Background slices are numbers 3-31 (amplitude

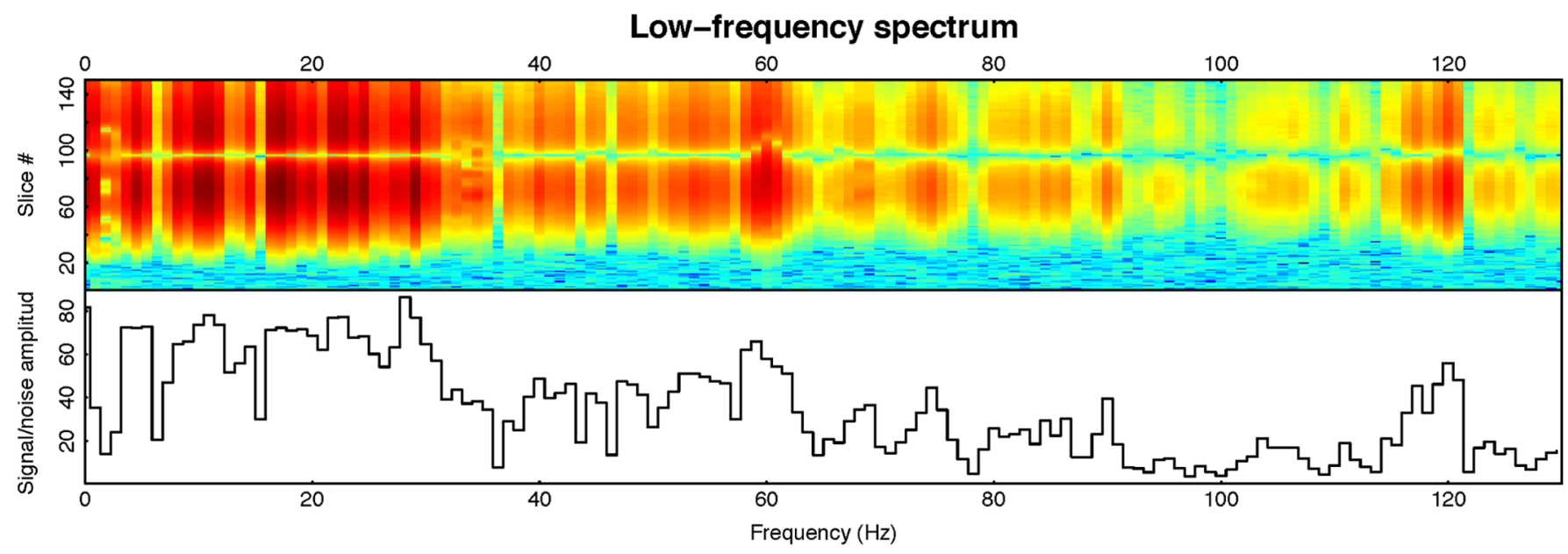

Tune spectrum

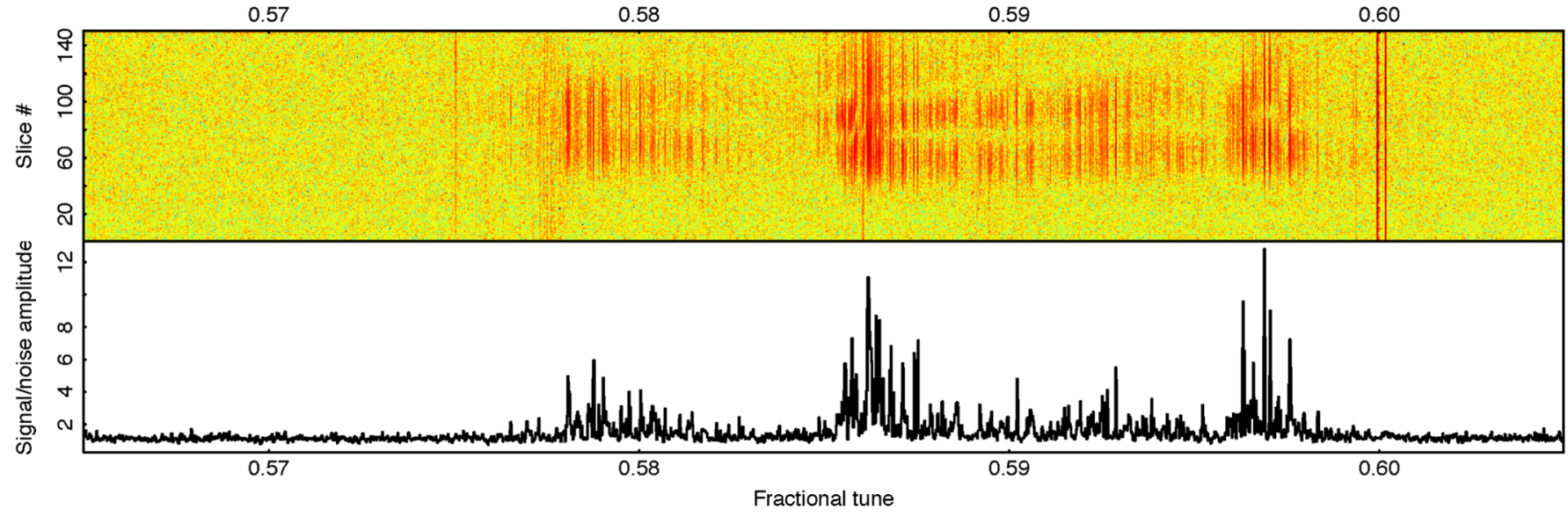

FIG. 5. Example of frequency spectra for antiprotons from data taken during Store 7754. Two selected regions of the spectrum are shown: below $130 \mathrm{~Hz}$ (top two plots) and around $47.7 \mathrm{kHz} \times(1-0.585)=20 \mathrm{kHz}$ (bottom two plots). The color plots represent the Fourier amplitude (in logarithmic scale) vs frequency for each slice. The black traces are the average amplitudes of the signal slices divided by those of the background slices (described in the text). 
below $2 \%$ of range). The black-and-white 1-dimensional plots show the ratio between signal and background amplitudes. In the top plots of Fig. 5, one can appreciate the strength of the low-frequency components. The $60-\mathrm{Hz}$ power-line noise and its harmonics are also visible. The lines around 34 and $68 \mathrm{~Hz}$ are due to synchrotron oscillations leaking into the transverse spectrum. The bottom plots of Fig. 5 show the spectra of transverse coherent oscillations. The vertical lines present in all slices in the 2 dimensional plot, attributed to digitizer noise, are eliminated by taking the ratio between signal and background slices. One can also notice the small variance of the noise level compared to the amplitude of the signal peaks.

In the 2-dimensional plots of Fig. 5, one may notice patterns in the oscillation amplitude as a function of position along the bunch. These may be an artifact of the imperfect synchronization between the $A$ and $B$ signals, but they may also be related to the physical nature of the coherent modes (i.e., rigid vs soft bunch, head-on vs long range). The phase of the oscillations as a function of frequency and bunch number may also provide physical insight. These aspects are not covered in the present analysis.

\section{RESULTS}

Transverse coherent mode spectra were measured for both proton and antiproton bunches under a wide range of experimental conditions. A few representative examples are discussed in this section. The machine and beam parameters relevant to the results presented below are collected in Table I.

Repeatability of the measurement was tested with antiprotons towards the end of Store 7719, when lattice tunes were kept constant and the beam-beam parameter was varying slowly. Figure 6 shows the results of 10 consecutive measurement cycles on antiproton bunches A13 and A14. The frequency resolution of single modes is high (52 488 turns were analyzed in this case). One may note the stability of the frequencies and the different distribution of amplitudes between the two bunches. The amplitude of each mode shows some variability, as it depends on the timing of the excitation and its duration, which were manually controlled.

At injection $(150 \mathrm{GeV})$ during Store 7706 , while antiprotons were being loaded into the machine on a separate orbit, the proton signal showed peaks at the injection lattice tunes $(0.585$ in the horizontal plane and 0.580 in the vertical one), even without excitation (Fig. 7, top). At collisions, it was usually necessary to apply the excitation to see a signal. When the excitation was applied, the signal was enhanced by at least a factor 10 and the pattern of transverse coherent beam-beam modes appeared (Fig. 7, center and bottom). The inset at the bottom of Fig. 7 shows that the frequency resolution is high enough to separate individual modes.

The amplitude of the signal without excitations and a comparison with the low-frequency beam jitter allows one to estimate the absolute magnitude of the coherent oscillations. For instance, if one takes a typical signal-tobackground ratio of 80 for the low-frequency motion, which corresponds to an amplitude of $25 \mu \mathrm{m}$, one obtains an amplitude of $60 \mathrm{~nm}$ for a signal-to-background ratio of 1.2 at the frequencies of interest. This translates into $20 \mathrm{~nm}$ for the average amplitude function of $75 \mathrm{~m}$ around the Tevatron ring (see also Ref. [34]). This is to be taken as a rough estimate, as the signal is often below the detection limit and it varies from store to store.

TABLE I. Summary of experimental conditions: instantaneous luminosity, $\mathcal{L}$; average number of protons and antiprotons per bunch, $N^{p}$ and $N^{a}$; average transverse emittances from the synchrotron-light monitor (95\%, normalized), $\varepsilon_{x}^{p}, \varepsilon_{y}^{p}$, $\varepsilon_{x}^{a}$, and $\varepsilon_{y}^{a}$; average longitudinal emittances, $\varepsilon_{z}^{p}$ and $\varepsilon_{z}^{a}$ (1 standard deviation); average incoherent tunes from the 1.7-GHz Schottky detector, $Q_{x}^{p}, Q_{y}^{p}, Q_{x}^{a}$, and $Q_{y}^{a}$; calculated linear beam-beam parameters per interaction point, $\xi_{x}^{p}, \xi_{y}^{p}, \xi_{x}^{a}$, and $\xi_{y}^{a}$.

\begin{tabular}{|c|c|c|c|c|c|c|c|c|c|c|c|c|c|c|c|c|c|c|c|}
\hline Store & Date & Time & $\begin{array}{c}\mathcal{L} 10^{32} / \\
\left(\mathrm{cm}^{2} \mathrm{~s}\right)\end{array}$ & $\begin{array}{c}N^{p} \\
10^{11}\end{array}$ & $\begin{array}{c}N^{a} \\
10^{11}\end{array}$ & $\begin{array}{c}\varepsilon_{x}^{p} \\
\mu \mathrm{m}\end{array}$ & $\begin{array}{c}\varepsilon_{y}^{p} \\
\mu \mathrm{m}\end{array}$ & $\begin{array}{c}\varepsilon_{x}^{a} \\
\mu \mathrm{m}\end{array}$ & $\begin{array}{c}\varepsilon_{y}^{a} \\
\mu \mathrm{m}\end{array}$ & $\begin{array}{c}\varepsilon_{z}^{p} \\
\mathrm{eVs}\end{array}$ & $\begin{array}{c}\varepsilon_{z}^{a} \\
\mathrm{eVs}\end{array}$ & $Q_{x}^{p}$ & $Q_{y}^{p}$ & $Q_{x}^{a}$ & $Q_{y}^{a}$ & $\xi_{x}^{p}$ & $\xi_{y}^{p}$ & $\xi_{x}^{a}$ & $\xi_{y}^{a}$ \\
\hline 7679 & 15 Mar 2010 & $16: 00$ & 3.350 & 2.891 & 0.8619 & 16.9 & 23.5 & 7.9 & 8.7 & 3.29 & 3.27 & 0.6038 & 0.5914 & 0.6175 & 0.5981 & 0.0078 & 0.0074 & 0.0115 & 0.0098 \\
\hline 7706 & 25 Mar 2010 & $15: 13$ & 3.500 & 2.953 & 0.9117 & 15.9 & 22.1 & 8.8 & 8.8 & 3.29 & 2.96 & 0.6082 & 0.6138 & 0.6235 & 0.6126 & 0.0076 & 0.0076 & 0.0125 & 0.0106 \\
\hline 7706 & 25 Mar 2010 & $16: 22$ & 2.670 & 2.756 & 0.8144 & 17.2 & 23.7 & 8.7 & 9.7 & 3.84 & 4.03 & 0.5910 & 0.5904 & 0.5876 & 0.5860 & 0.0067 & 0.0063 & 0.0108 & 0.0092 \\
\hline 7711 & 26 Mar 2010 & 12:09 & 1.430 & 2.822 & 0.5842 & 22.5 & 31.3 & 11.6 & 13.8 & 4.71 & 4.59 & 0.5888 & 0.5889 & 0.5868 & 0.5868 & 0.0035 & 0.0032 & 0.0084 & 0.0072 \\
\hline 7719 & 1 Apr 2010 & $11: 58$ & 0.810 & 2.272 & 0.5319 & 23.8 & 35.6 & 18.3 & 18.3 & 6.33 & 5.52 & 0.5880 & 0.5881 & 0.5875 & 0.5866 & 0.0021 & 0.0021 & 0.0063 & 0.0051 \\
\hline 7724 & 2 Apr 2010 & $15: 38$ & 2.950 & 2.791 & 0.8475 & 17.0 & 24.7 & 9.2 & 9.3 & 3.57 & 3.21 & 0.5901 & 0.5904 & 0.5908 & 0.5969 & 0.0067 & 0.0067 & 0.0109 & 0.0091 \\
\hline 7724 & 2 Apr 2010 & 16:07 & 2.690 & 2.752 & 0.8197 & 17.4 & 25.4 & 9.5 & 9.8 & 3.73 & 3.39 & 0.5887 & 0.5892 & 0.5857 & 0.5907 & 0.0063 & 0.0062 & 0.0105 & 0.0087 \\
\hline 7754 & 21 Apr 2010 & 16:09 & 3.510 & 2.856 & 0.9428 & 15.0 & 23.3 & 7.7 & 9.4 & 3.31 & 3.52 & 0.5978 & 0.5908 & 0.6163 & 0.6096 & 0.0085 & 0.0077 & 0.0124 & 0.0100 \\
\hline 7754 & 21 Apr 2010 & $16: 24$ & 3.200 & 2.810 & 0.9236 & 15.1 & 23.1 & 7.9 & 9.4 & 4.41 & 3.64 & 0.5901 & 0.5899 & 0.5973 & 0.6034 & 0.0082 & 0.0075 & 0.0122 & 0.0099 \\
\hline 7754 & 21 Apr 2010 & $16: 45$ & 2.970 & 2.769 & 0.9008 & 15.4 & 23.8 & 8.6 & 9.8 & 4.43 & 3.72 & 0.5924 & 0.5897 & 0.5864 & 0.5900 & 0.0074 & 0.0070 & 0.0117 & 0.0095 \\
\hline 7754 & 21 Apr 2010 & $17: 10$ & 2.730 & 2.737 & 0.8806 & 15.8 & 24.4 & 9.0 & 10.0 & 4.50 & 3.85 & 0.5890 & 0.5889 & 0.5867 & 0.5872 & 0.0070 & 0.0066 & 0.0113 & 0.0091 \\
\hline 7754 & 21 Apr 2010 & 21:41 & 1.570 & 2.554 & 0.7333 & 18.6 & 29.1 & 12.3 & 13.7 & 5.54 & 4.82 & 0.5883 & 0.5885 & 0.5867 & 0.5863 & 0.0043 & 0.0040 & 0.0089 & 0.0071 \\
\hline 7754 & 22 Apr 2010 & $10: 24$ & 0.670 & 2.241 & 0.5072 & 23.8 & 36.8 & 19.6 & 20.7 & 6.85 & 6.04 & 0.5882 & 0.5887 & 0.5871 & 0.5877 & 0.0019 & 0.0018 & 0.0061 & 0.0049 \\
\hline 7893 & 15 Jun 2010 & $13: 26$ & 0.055 & 2.703 & 0.4600 & 25.9 & 36.0 & 19.6 & 20.1 & 3.86 & 2.78 & 0.5849 & 0.5852 & 0.5639 & 0.5628 & 0.0017 & 0.0017 & 0.0070 & 0.0060 \\
\hline
\end{tabular}




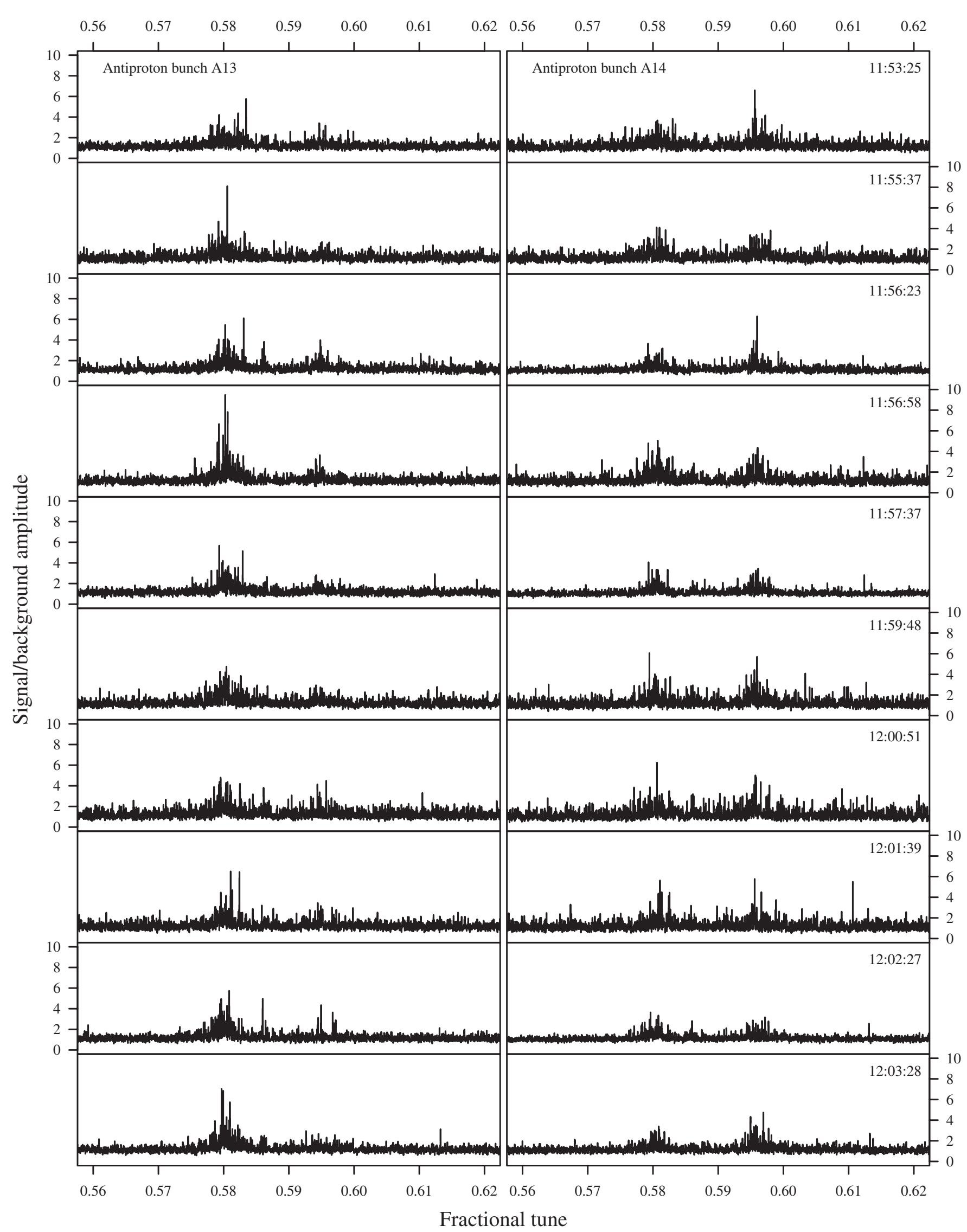

FIG. 6. Repeated measurements on 2 antiproton bunches during Store 7719 . 


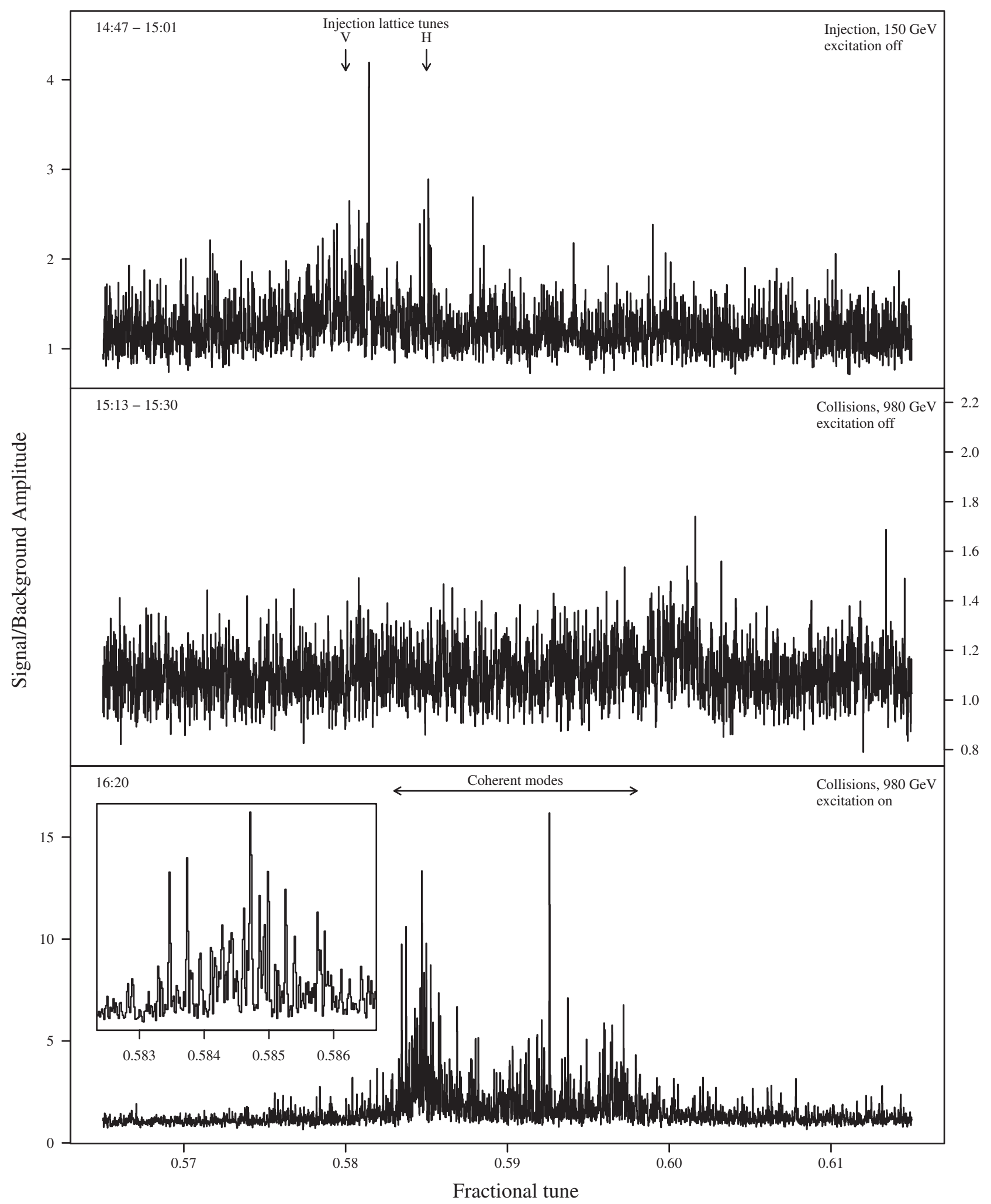

FIG. 7. Vertical coherent modes for one proton bunch during Store 7706, at injection and at collisions.

Another example of signals measured without excitation is shown in Fig. 8. Data were collected during Store 7679 for antiproton bunch A25 at top energy after the low-beta squeeze, after initiating collisions, and after scraping the halo. One can see slowly damped oscillations around $Q=0.6$ and their synchrotron sidebands.

During Store 7724, the signals of protons and antiprotons were compared (Fig. 9). For equal beam intensities, 


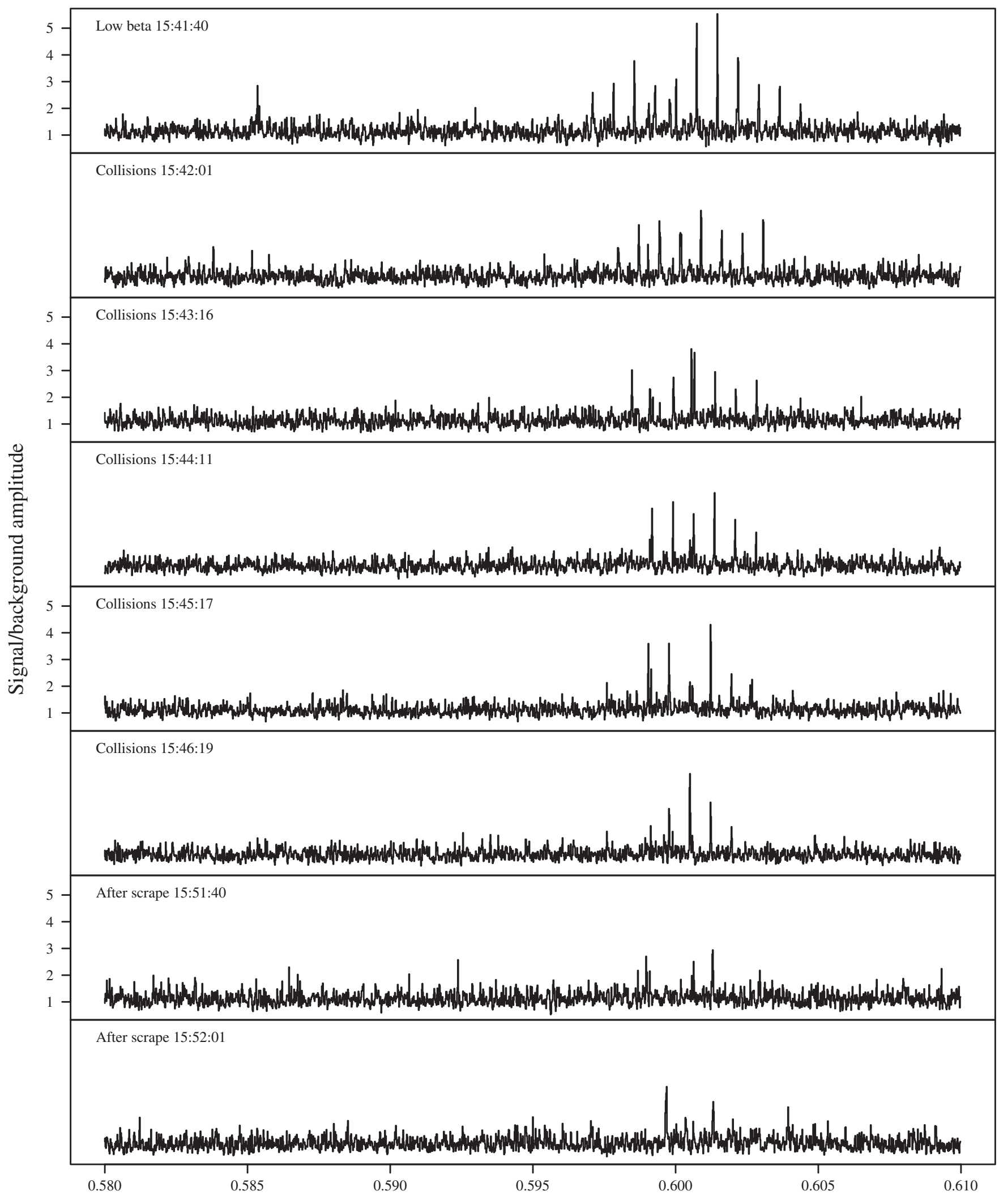

Fractional tune

FIG. 8. Vertical coherent modes for antiproton bunch A25 during Store 7679 with no excitation, from the low-beta squeeze to after halo scraping. 


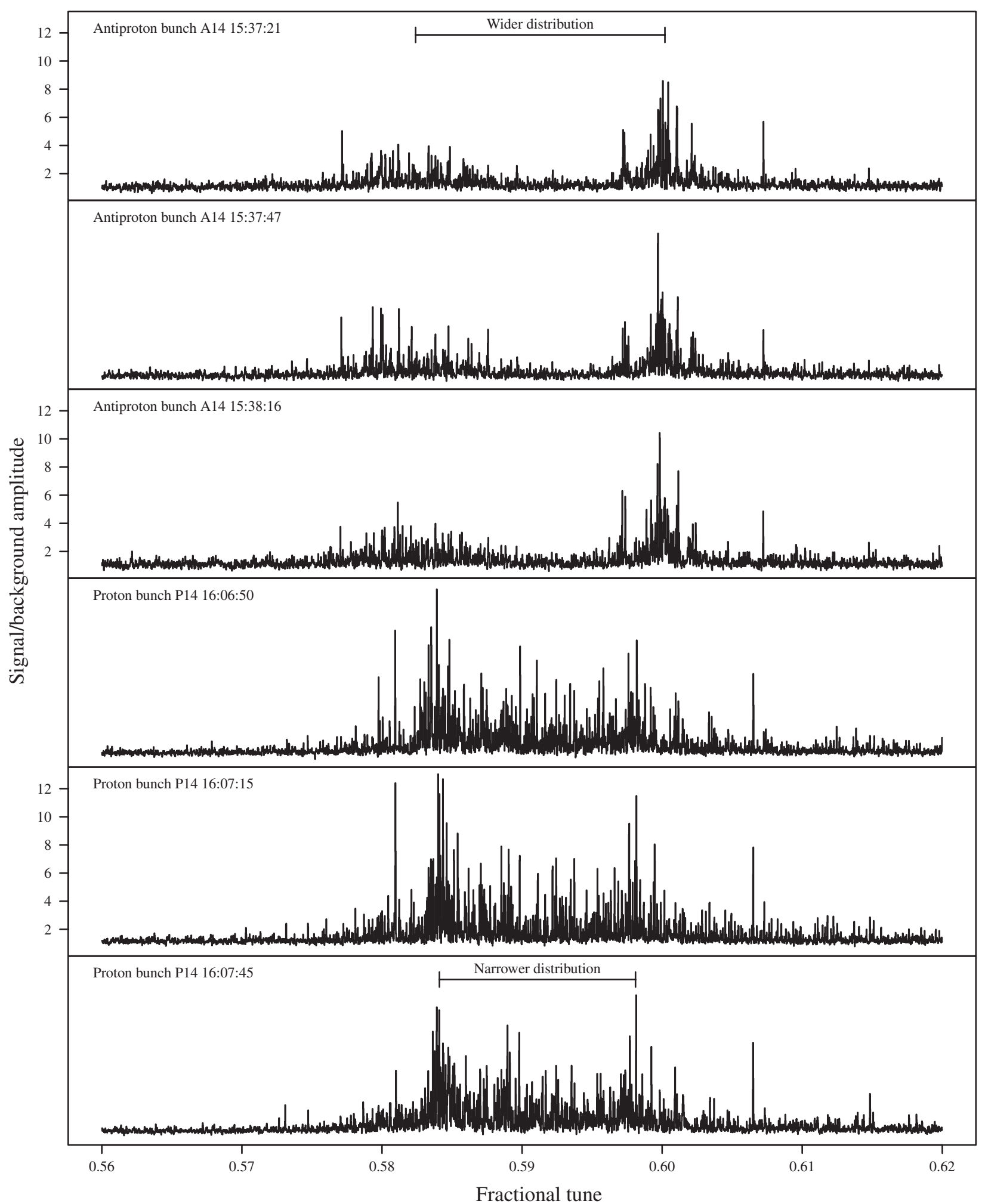

FIG. 9. Comparison of antiproton and proton vertical coherent modes during Store 7724.

emittances, and tunes, one would expect to observe the same modes in both beams. The elapsed time between the two sets of measurements was necessary to swap cables and re-equalize the signals. The structure of the spectra is similar, but the antiproton distribution is wider. From this, one can infer that the antiproton beam-beam parameter was larger. The calculated values were $\xi_{y}^{a}=0.009$ and $\xi_{y}^{p}=$ 0.006 at each of the 2 collision points. 


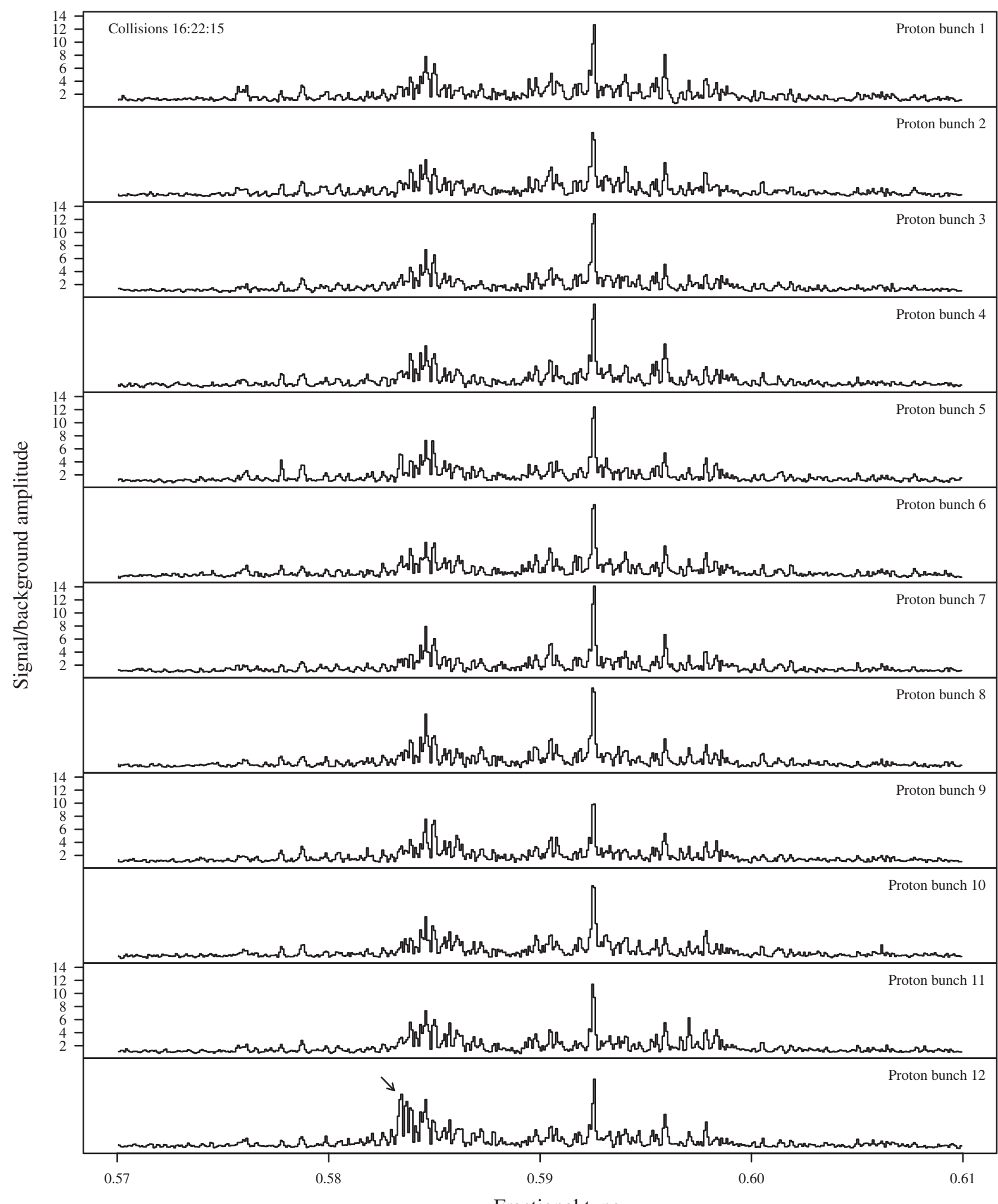

Fractional tune

FIG. 10. Vertical coherent modes for all proton bunches in a train during Store 7706 .

An example of bunch-by-bunch measurements for protons during Store 7706 is shown in Fig. 10. The signal from all 12 bunches in a train was recorded for 12382 turns. All bunches show very similar spectra except for P12, for which stronger lower modes are present (indicated by the arrow), probably due to long-range interactions.

Figure 11 shows an example of the response of the proton coherent mode spectra to changes in the vertical 


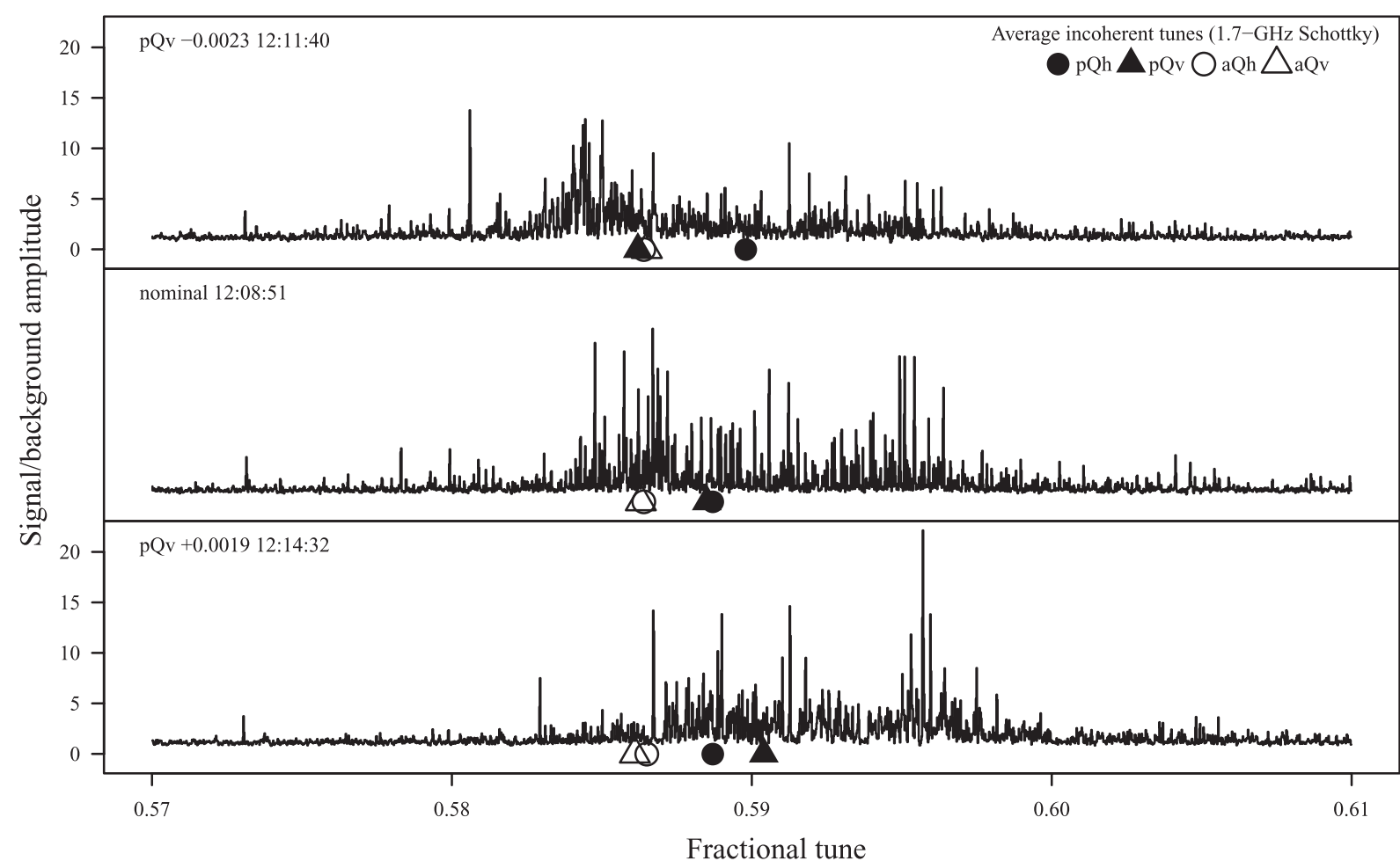

FIG. 11. Response of vertical coherent modes for proton bunch P11 to lattice tune changes during Store 7711.

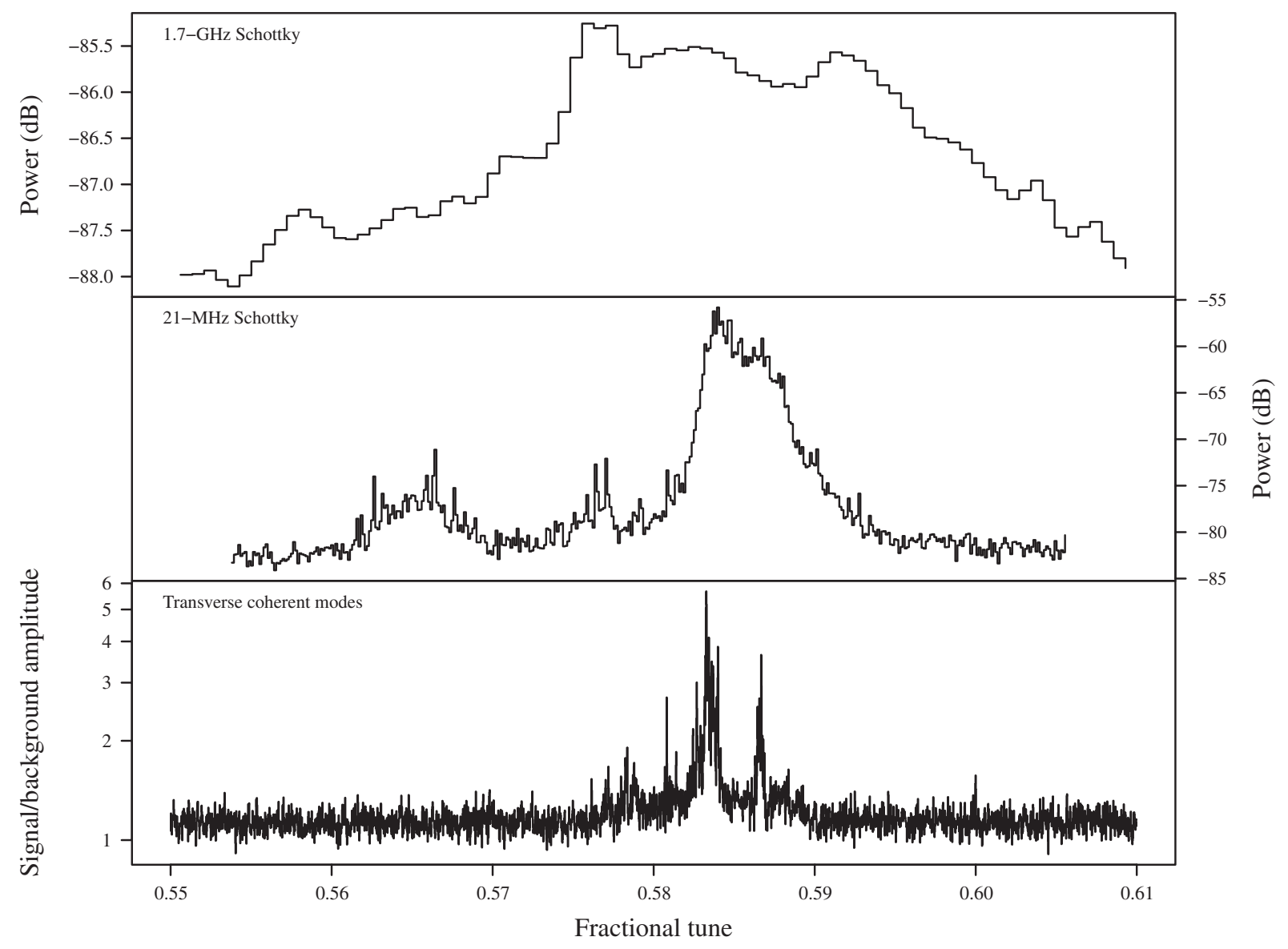

FIG. 12. Comparison of Schottky and coherent spectra during dedicated 3-on-3 Store 7893. 
proton lattice tune during Store 7711 . The lattice setting of $Q_{y}^{p}$ was changed from -0.0023 to +0.0019 with respect to the nominal value. The average incoherent tunes measured by the $1.7-\mathrm{GHz}$ Schottky detectors are also shown for comparison (circles and triangles). In this experiment, the vertical beam-beam parameter for protons $\left(\xi_{y}^{p}=0.003\right)$ was about twice as small as the one for antiprotons.

A special store with only 3 equally spaced proton bunches colliding with 3 antiproton bunches was studied

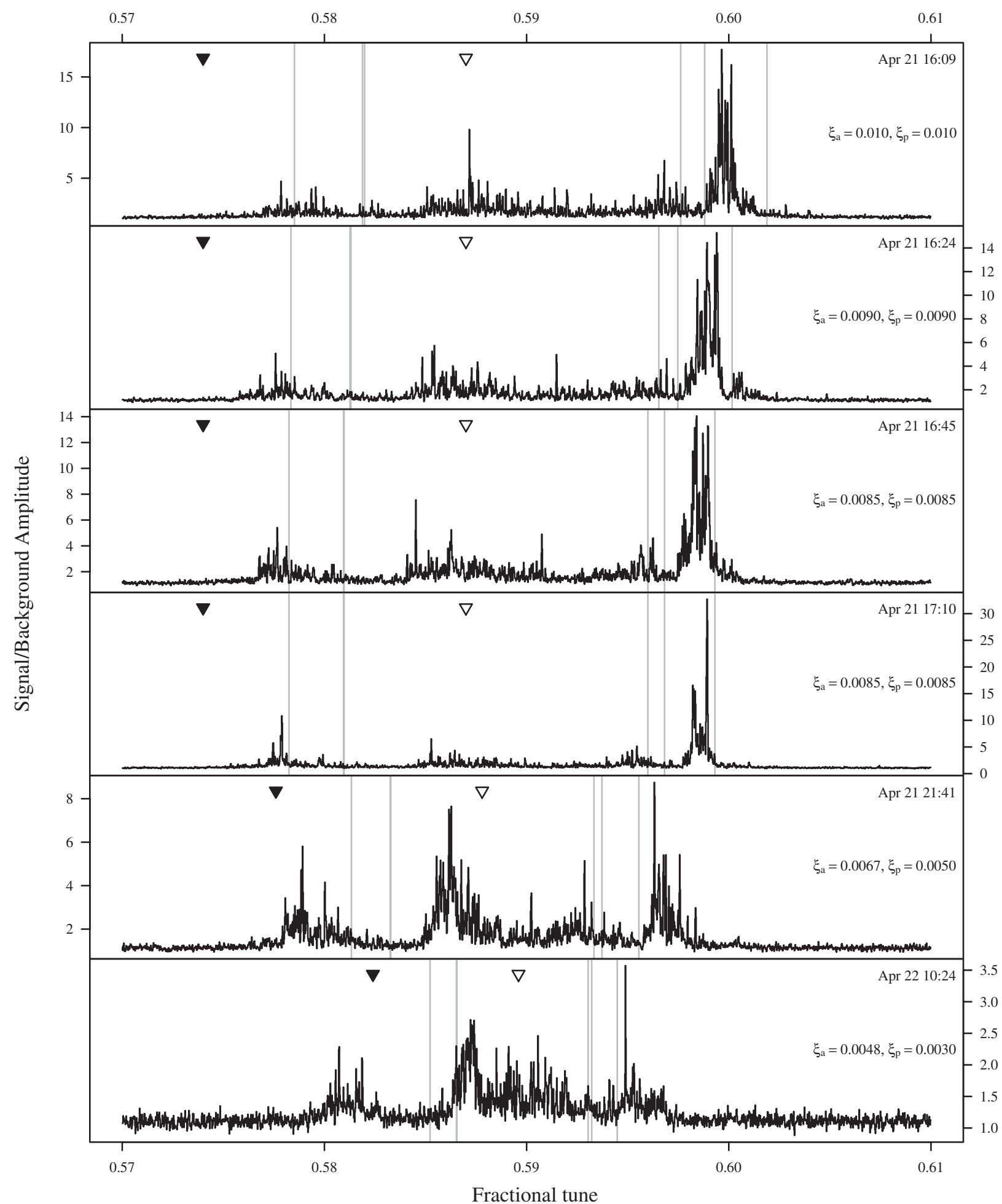

FIG. 13. Evolution of vertical coherent beam-beam modes for antiproton bunch A13 during the course of Store 7754 . 
for beam-beam compensation purposes (Store 7893). During this store, the signal from 3 systems was recorded: the 1.7-GHz Schottky monitor, the 21-MHz Schottky monitor, and the coherent mode detector described in this paper (Fig. 12). Unfortunately, transverse beam sizes were large, making the beam-beam parameter quite small. However, this measurement illustrates the unique features of each system. The 1.7-GHz Schottky monitor has bunch-bybunch capability and good separation of the proton and antiproton signals. It is routinely used to report the average tune of each beam every minute. Because it operates at a large harmonic number $(h=35631)$, the sidebands contain a large number of synchrotron satellites and their widths are dominated by the momentum spread of the beam. The 21-MHz Schottky system has better frequency resolution, but it cannot distinguish protons from antiprotons and it is not gated to individual bunches. The coherent mode detector has high-frequency resolution and fast bunch-by-bunch response. It currently requires excitation of the beam, but this limitation can be overcome by automatically filtering and equalizing the signals to extend its dynamic range. The interpretation of the spectra for large beam-beam parameters, high number of bunches, and complex collision patterns can also be considered a limitation.

An illustration of the evolution of transverse coherent modes over a complete store is shown in Fig. 13 for vertical antiproton oscillations. As expected, one can see that as the beam-beam force weakens, the spread in coherent modes decreases. Over the course of a store, the lattice tunes need to be periodically adjusted to keep the average incoherent tune close to the desired working point. The bare lattice tune for antiprotons (black triangles) and for protons (empty triangles) is estimated from the machine settings and their calibration. The vertical gray lines represent the prediction of the simplified model presented in Sec. II using the estimated bare lattice tunes and the beam-beam parameters calculated from the measured beam intensities and synchrotron-light emittances. Except for the last two measurements, which may be affected by the evolving linear coupling and by a slight miscalibration of the tune settings, one can see that the estimated lattice tune lies below the first group of eigenmodes, and that the predicted eigenfrequencies are close to the measured peaks. Obviously, the measured spectra are richer than those predicted by the simplified model, and a complete explanation requires a more detailed description of the beam dynamics, such as the one found in Ref. [21] based on a 3-dimensional strongstrong particle-in-cell beam-beam code.

\section{CONCLUSIONS}

A system was developed to measure the spectra of coherent beam-beam oscillations of individual bunches in the Tevatron. It is based on the analysis of the digitized signal from a single beam-position monitor. It requires applying band-limited noise to the beam, but an extension of its dynamic range should be possible, if needed, so as to operate without excitation.

The device has a response time of a few seconds, a frequency resolution of $1.6 \times 10^{-5}$ in fractional tune, and it is sensitive to oscillation amplitudes of $60 \mathrm{~nm}$. In terms of sensitivity, resolution, and background level, it provides a very clean measurement of coherent oscillations in hadron machines. The system complements Schottky detectors and beam transfer function measurements as a diagnostic tool for tunes, tune spreads, and beam-beam effects.

It was confirmed that coherent oscillations in the Tevatron are stable, probably thanks to the different intensities of the two beams, their tune separation, and chromaticity. The average amplitude of the oscillations around the ring was estimated to be of the order of $20 \mathrm{~nm}$.

A simplified collision model was used to calculate mode eigenfrequencies and to show their dependence on the beam-beam coupling. It was compared with observations made over the course of a collider store, as the strength of the beam-beam force decreased with time.

Spectra were acquired at different tune separations, beam-beam parameters, and collision schemes to provide an experimental basis for beam-beam numerical codes.

\section{ACKNOWLEDGMENTS}

The authors would like to thank V. Kamerdzhiev (Forschungszentrum Jülich, Germany), F. Emanov (Budker Institute for Nuclear Physics, Novosibirsk, Russia), Y. Alexahin, B. Fellenz, V. Lebedev, G. Saewert, V. Scarpine, A. Semenov, and V. Shiltsev (Fermilab) for their help and insights. Fermilab is operated by Fermi Research Alliance, LLC under Contract No. DE-AC0207CH11359 with the United States Department of Energy.

[1] A. Piwinski, IEEE Trans. Nucl. Sci. 26, 4268 (1979).

[2] T. Ieiri, T. Kawamoto, and K. Hirata, Nucl. Instrum. Methods Phys. Res., Sect. A 265, 364 (1988).

[3] E. Keil, K. Cornelis, and K. Hirata, in Proceedings of the 15th International Conference on High Energy Accelerators, Hamburg, Germany), edited by J. Rossbach (World Scientific, Singapore, 1993), p. 1106 [http://cdsweb.cern.ch/record/239617].

[4] I. N. Nesterenko, E. A. Perevedentsev, and A. A. Valishev, Phys. Rev. E 65, 056502 (2002).

[5] J.P. Koutchouk, CERN Report No. ISR-OP/JPK-svw, 1982 [http://cdsweb.cern.ch/record/1131719].

[6] J. P. Koutchouk, CERN Report No. ISR-OP/JPK-bm, 1982 [http://cdsweb.cern.ch/record/1131796].

[7] W. Fischer, L. Ahrens, M. Bai, M. Blaskiewicz, P. Cameron, R. Michnoff, F. Pilat, V. Ptitsyn, T. Sen, S. Tepikian, D. Trbojevic, M. Vogt, and J. van Zeijts, BNL Report No. C-AD/AP/75, 2002.

[8] W. Fischer, M. Blaskiewicz, J. M. Brennan, P. Cameron, R. Connolly, C. Montag, S. Peggs, F. Pilat, V. Ptitsyn, S. Tepikian, D. Trbojevic, and J. van Zeijts, in Proceedings of 
the 2003 Particle Accelerator Conference, Portland, OR (IEEE, Piscataway, NJ, 2003), p. 135.

[9] T. Pieloni, Ph.D. thesis, École Polytechnique Fédérale de Lausanne, Switzerland, 2008.

[10] Y. Alexahin, Part. Accel. 59, 43 (1998).

[11] V. Shiltsev, V. Danilov, D. Finley, and A. Sery, Phys. Rev. ST Accel. Beams 2, 071001 (1999).

[12] V. Shiltsev, Y. Alexahin, K. Bishofberger, V. Kamerdzhiev, V. Parkhomchuk, V. Reva, N. Solyak, D. Wildman, X.-L. Zhang, and F. Zimmermann, New J. Phys. 10, 043042 (2008).

[13] V. Shiltsev, K. Bishofberger, V. Kamerdzhiev, S. Kozub, M. Kufer, G. Kuznetsov, A. Martinez, M. Olson, H. Pfeffer, G. Saewert, V. Scarpine, A. Seryi, N. Solyak, V. Sytnik, M. Tiunov, L. Tkachenko, D. Wildman, D. Wolff, and X.-L. Zhang, Phys. Rev. ST Accel. Beams 11, 103501 (2008).

[14] A. Valishev and G. Stancari, in Proceedings of the 2011 Particle Accelerator Conference, New York, NY (IEEE, Piscataway, NJ, 2011), MOODN1, p. 67.

[15] R.E. Meller and R. H. Siemann, IEEE Trans. Nucl. Sci. 28, 2431 (1981).

[16] K. Yokoya and H. Koiso, Part. Accel. 27, 181 (1990).

[17] W. Herr, M. P. Zorzano, and F. Jones, Phys. Rev. ST Accel. Beams 4, 054402 (2001).

[18] Y. Alexahin, Nucl. Instrum. Methods Phys. Res., Sect. A 480, 253 (2002).

[19] T. Pieloni and W. Herr, in Proceedings of the 2005 Particle Accelerator Conference, Knoxville, TN (IEEE, Piscataway, NJ, 2005), p. 4030.

[20] J. Qiang, M. A. Furman, R. D. Ryne, W. Fischer, and K. Ohmi, Nucl. Instrum. Methods Phys. Res., Sect. A 558, 351 (2006).

[21] E. G. Stern, J. F. Amundson, P. G. Spentzouris, and A. A. Valishev, Phys. Rev. ST Accel. Beams 13, 024401 (2010).

[22] J.-P. Carneiro, V. Lebedev, V. Scarpine, A. Semenov, V. Shiltsev, and R. Webber, Fermilab Report Beams-doc1911-v1 [http://beamdocs.fnal.gov].

[23] A. Semenov, J.-P. Carneiro, V. Kamerdzhiev, and V. Lebedev, in Proceedings of the 2007 Particle
Accelerator Conference, Albuquerque, NM (IEEE, Piscataway, NJ, 2007), p. 3877.

[24] V. Kamerdzhiev, V. Lebedev, and A. Semenov, in Proceedings of the 2008 Beam Instrumentation Workshop (BIW08), Tahoe City, California (2008), p. 300 [http://www.jacow.org].

[25] A. Valishev, F. Emanov, V. Kamerdzhiev, and V. Lebedev, in Proceedings of the 2008 European Particle Accelerator Conference (EPAC08), Genoa, Italy (EPS-AG, Genoa, Italy, 2008), p. 3158.

[26] G. Stancari, A. Valishev, and A. Semenov, in Proceedings of the 2010 Beam Instrumentation Workshop (BIW10), Santa Fe, New Mexico (2010), p. 363 [http://www.lanl .gov/conferences/biw10].

[27] K. Yokoya, Phys. Rev. ST Accel. Beams 3, 124401 (2000).

[28] R Development Core Team, $R$ : A language and environment for statistical computing ( $\mathrm{R}$ Foundation for Statistical Computing, Vienna, Austria, 2010) [http:// www.R-project.org].

[29] B. Baklakov, T. Bolshakov, A. Chupyra, A. Erokhin, P. Lebedev, V. Parkhomchuk, Sh. Singatulin, J. Lach, and V. Shiltsev, in Proceedings of the 1999 Particle Accelerator Conference, New York, NY (IEEE, Piscataway, NJ, 1999), p. 1387.

[30] W. H. Press, S. A. Teukolsky, W. T. Vetterling, and B.P. Flannery, Numerical Recipes: The Art of Scientific Computing (Cambridge University Press, Cambridge, England, 2007), 3rd ed.

[31] N. Kurosawa, K. Maruyama, H. Kobayashi, H. Sugawara, and K. Kobayashi, IEEE Proc. Instrum. Meas. Tech. Conf. 2, 763 (2000).

[32] J. Elbornsson, F. Gustafsson, and J.-E. Eklund, IEEE Trans. Signal Process. 53, 1413 (2005).

[33] P. Fong, A. Teruya, and M. Lowry, in Proceedings of the Instrumentation and Measurement Technology Conference (IMTC 2005) (IEEE, Piscataway, NJ, 2005), p. 417 [http:// dx.doi.org/10.1109/IMTC.2005.1604149].

[34] V. Shiltsev, in Proceedings of the 2011 Particle Accelerator Conference, NY, USA (Ref. [14]), THP067. 